\title{
Ceric Ion Loaded MCM-41 Catalyzed Synthesis of Substituted Mono- and Bis-dihydropyrimidin-2(1H)-ones
}

\author{
Pullar Vadivel, ${ }^{1}$ Rathinam Ramesh, ${ }^{2}$ and Appaswami Lalitha ${ }^{2}$ \\ ${ }^{1}$ Department of Chemistry, Salem Sowdeswari College, Salem, Tamil Nadu 636010, India \\ ${ }^{2}$ Department of Chemistry, Periyar University, Periyar Palkalai Nagar, Salem, Tamil Nadu 636011, India \\ Correspondence should be addressed to Appaswami Lalitha; lalitha2531@yahoo.co.in
}

Received 30 May 2013; Accepted 29 October 2013

Academic Editor: Adel A. Ismail

Copyright (c) 2013 Pullar Vadivel et al. This is an open access article distributed under the Creative Commons Attribution License, which permits unrestricted use, distribution, and reproduction in any medium, provided the original work is properly cited.

\begin{abstract}
An effective one-pot three-component reaction of aromatic aldehydes with 1,3-diketone and urea or thiourea under solvent-free condition leads to the formation of mono- and bis-dihydropyrimidin-2-(1H)-ones using Ce-MCM-41 as a recyclable solid acid catalyst. This method has several advantages like simple and easy work-up with shorter reaction time, reusability of catalyst, and high yields of Biginelli products.
\end{abstract}

\section{Introduction}

Multicomponent reactions (MCRs) have been received significantly as a valuable synthetic tool in the field of modern organic synthesis and drug discovery research due to their ability to synthesize target compounds with greater efficiency in single step operations of three or more different monofunctionalized reactants. Moreover, MCRs offer some distinct advantages including atom economy, structural variations, complexity of molecules, and simplicity over conventional step by step synthetic procedures [1-8].

Biginelli reaction is a well-known, simple, and straightforward method for the synthesis of 3,4-dihydropyrimidinones (DHPMs) which involves the three-component condensation of an aliphatic or aromatic aldehyde, $\beta$-ketoester, and urea or thiourea. The original reaction was first reported by Biginelli in 1893 catalyzed by mineral acids [9]. Different functionalized 3,4-DHPMs synthesized have exhibited a variety of pharmacological activities such as calcium channel modulation [10], mitotic kinesin Eg5 inhibition (monastrol) [3], antiviral [11], antibacterial, antifungal [12], and anticancer [13]. DHPMs are also used as starting materials for the synthesis of so called "superstatin" rosuvastatin, a selective and competitive inhibitor of HMG-CoA reductase [14], the enzyme responsible for the biosynthesis of cholesterol. Moreover, the 3,4-DHPM motif is present in many products isolated from natural material like several species of sponges.
Due to the wide range applications, several methods have been reported for the synthesis of dihydropyrimidinones that include the utilization of $\mathrm{BF}_{3} \cdot \mathrm{OEt}_{2} / \mathrm{CuCl}$ [15], lanthanide triflate [16], indium trichloride [17], vanadium (III) chloride [18], cupric chloride [19], LiBr [20], zirconium (IV) chloride [21], lithium perchlorate [22], and polymer-supported ytterbium (II) reagent [23] as well as Bronsted acids, such as $p$ toluenesulfonic acid [24], silica sulfuric acid [25], $\mathrm{KHSO}_{4}$ [26], and also solid acids like montmorillonite KSF [27], natural HEU-type zeolite [28], and HY-zeolite [29]. However, many of these reported methods suffer from drawbacks such as low yield of products, harsh reaction conditions and long experimental procedures, and toxic and costly catalysts. Therefore, there is a need to develop new catalysts which are easily available or prepared, cost-effective, recoverable, and environment friendly and almost all these requirements may be easily met by the supported reagents. In this paper, we wish to report for the first time a simple, facile, and highly efficient method for the synthesis of 3,4-dihydropyrimidin2 -ones in excellent yields by the three-component reaction of aldehydes, urea, and $\beta$-ketoester using Ce-MCM- 41 as a solid heterogeneous catalyst.

\section{Experimental}

2.1. Materials. All aldehydes, $\beta$-ketoesters, urea, and thiourea were purchased from commercial sources and used without 


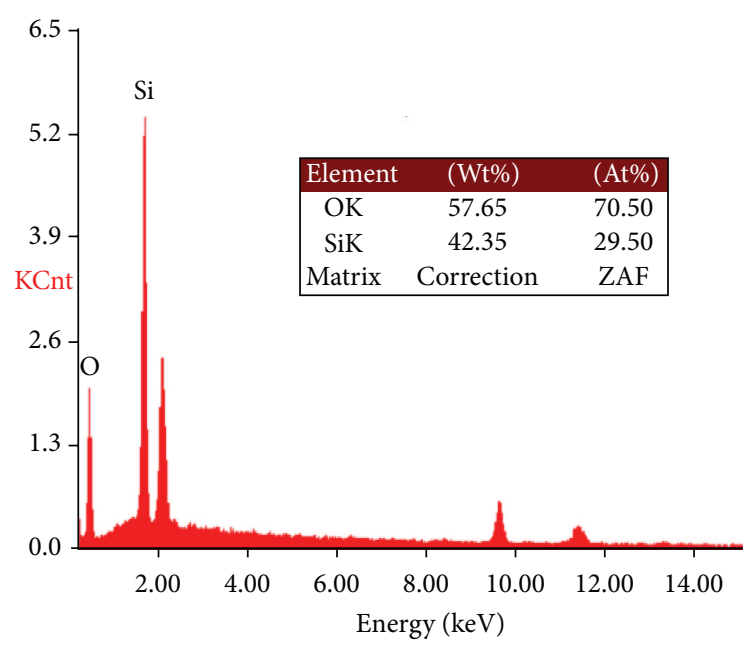

(a)

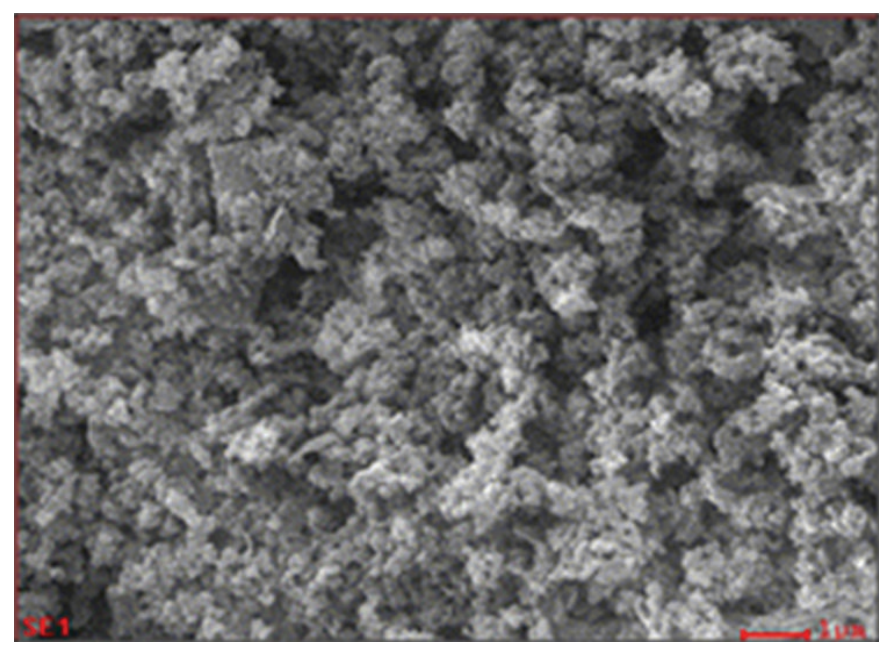

(b)

FIGURE 1: EDX and SEM image of MCM-41 material.

purification. All reactions were performed in a reaction vessel open to the atmosphere and monitored by thin layer chromatography (TLC). ${ }^{1} \mathrm{H}$ and ${ }^{13} \mathrm{C}$ NMR spectra were recorded on $400-\mathrm{MHz}$ Bruker spectrometer. Fourier transform infrared (FT-IR) spectra were obtained using $\mathrm{KBr}$ technique.

\subsection{Catalyst Preparation}

2.2.1. Synthesis of MCM-41. About $1.988 \mathrm{~g}$ of cetyl trimethyl ammonium bromide (CTAB, 98\%) was dissolved in $120 \mathrm{~mL}$ of water at room temperature. After complete dissolution, $8 \mathrm{~mL}$ of aqueous ammonia (32\% in water) was added and then $10 \mathrm{~mL}$ of tetraethyl orthosilicate (TEOS, 99\%) was added with vigorous stirring $(300 \mathrm{rpm})$. The hydrolysis of TEOS happened during the first $2 \mathrm{~min}$ at room temperature (the solution becomes milky and slurry forms) and the condensation of the mesostructured hybrid material was achieved after $2 \mathrm{~h}$ of reaction. The material was then filtered and allowed to dry under static air at $80^{\circ} \mathrm{C}$ for $12 \mathrm{~h}$. The mesoporous material was finally obtained by calcining the hybrid structure at $550^{\circ} \mathrm{C}$ for $5 \mathrm{~h}$.

2.2.2. Preparation of $C e(I V)$ Loaded MCM-41. $0.1 \mathrm{~g}$ of activated MCM- 41 was added into the acetone solution of $0.11 \mathrm{~g}$ of ceric ammonium nitrate (CAN). The yellow colored mixture was slowly evaporated to dryness with stirring. The yellow solid has been warmed slightly to make it completely dry. Then the solid was calcined for about $1 \mathrm{~h}$ to remove the nitrate ions (liberated as yellow fumes). After calcinations, the yellow colored powder was kept for activation in muffle furnace for $4 \mathrm{~h}$ yielding the catalyst with $20 \mathrm{~mol} \%$ of ceric ions. Various concentrations of ceric ion such as $10 \%, 20 \%, 30 \%$, and $40 \%$ were loaded on MCM-41 employing the above method.

\subsubsection{Characterization}

2.2.4. SEM and EDX Analysis. Figures 1, 2, 3, and 4 show the surface morphology and EDX analysis of MCM-41, 10,
20 , and $30 \mathrm{~mol} \%$ of ceric ion loaded MCM-41. The particle size of the Ce containing MCM-41 material was ranging from 200 to $350 \mathrm{~nm}$. Here, the size of the nanoparticles decreased compared to the particle size of normal MCM-41. This may be attributed to the addition of ceric ion onto MCM-41 which prevents the agglomeration of the particles. The EDX analysis result shows the presence of cerium, oxygen, and silicon at $21.59 \mathrm{wt} \%, 41.87 \mathrm{wt} \%$, and $36.54 \mathrm{wt} \%$, respectively, which proves the formation of ceric ion functionalized MCM-41. SEM micrographs for the calcined blank MCM-41 along with the $10 \mathrm{~mol} \%$ Ce-MCM-41, $20 \mathrm{~mol} \%$ Ce-MCM-41, and $30 \mathrm{~mol} \% \mathrm{Ce}-\mathrm{MCM}-41$ composites are shown in Figures $1,2,3$, and 4, respectively. Spherical particles formation was observed for the MCM-41 material, and this spherical morphology was preserved for the composite materials.

\subsection{General Synthetic Procedure for the Preparation of DHPM} Derivatives (4a-v). A mixture of aldehyde ( $1 \mathrm{mmol}), \beta$ ketoester $(1 \mathrm{mmol})$, urea or thiourea $(1.5 \mathrm{mmol})$, and CeMCM-41 (100 mg) was finely ground and heated at $80^{\circ} \mathrm{C}$ for specified time. After the reaction, the crude product from the reaction mixture was dissolved in hot ethanol and the catalyst was separated by filtration. The filtrate was suspended in water to precipitate the resulting product. The solid product was then crystallized from hot ethanol.

2.4. General Synthetic Procedure for the Preparation of Bis-DHPM Derivatives (5a-d). A mixture of bis-aldehyde $(1 \mathrm{mmol}), \beta$-ketoester $(2 \mathrm{mmol})$, urea or thiourea $(3 \mathrm{mmol})$, and Ce-MCM-41 (100 mg) was finely ground and heated at $80^{\circ} \mathrm{C}$ for specified time. After the reaction, the pure product can be discovered by the above procedure.

2.5. Product Characterization. All the mono- and bisdihydropyridimin-2-one or pyrimidine-2-thione derivatives are known and were characterized by their m.p., IR, ${ }^{1} \mathrm{H}$, and ${ }^{13} \mathrm{C}-\mathrm{NMR}$ spectral data. 


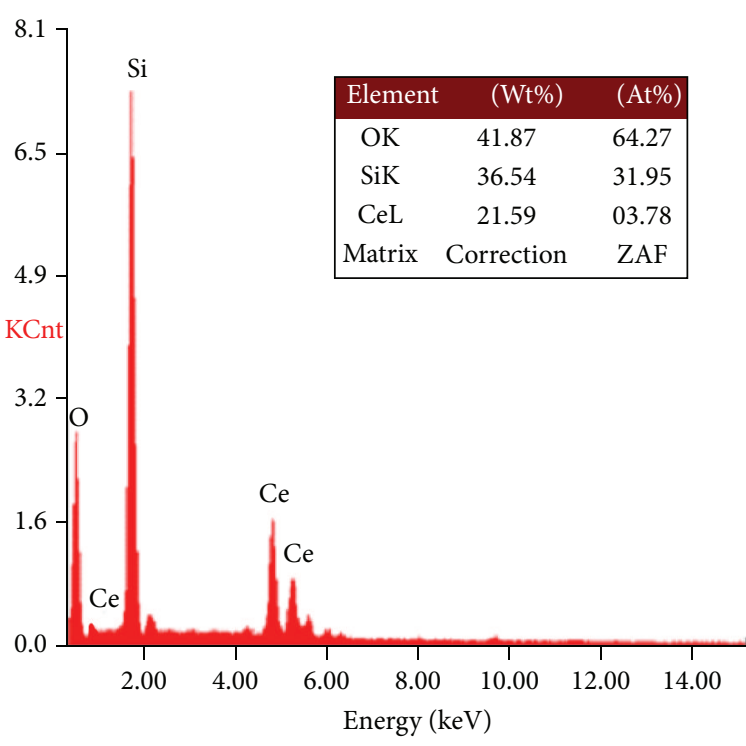

(a)

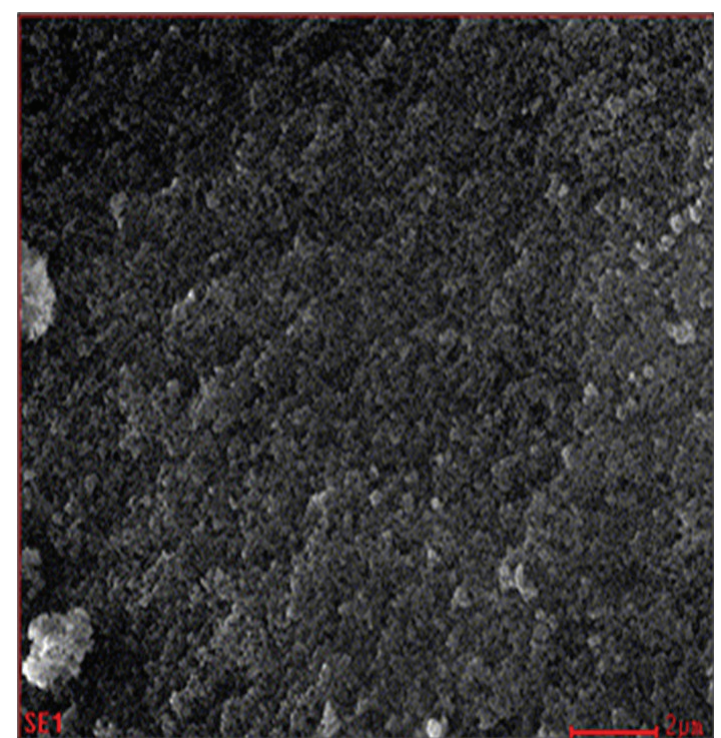

(b)

Figure 2: EDX and SEM image of 10 mol\% Ce loaded MCM-41 material.

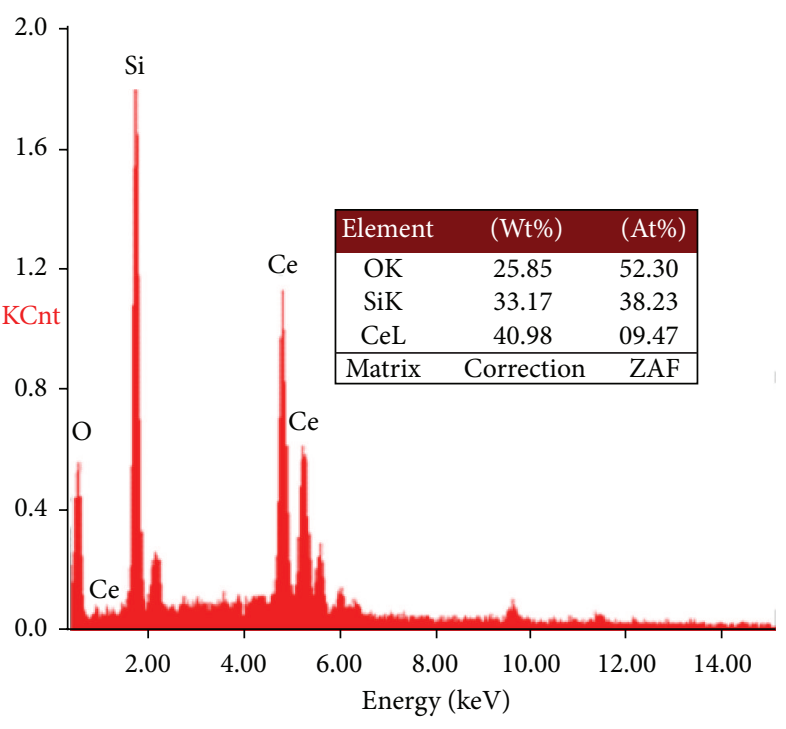

(a)

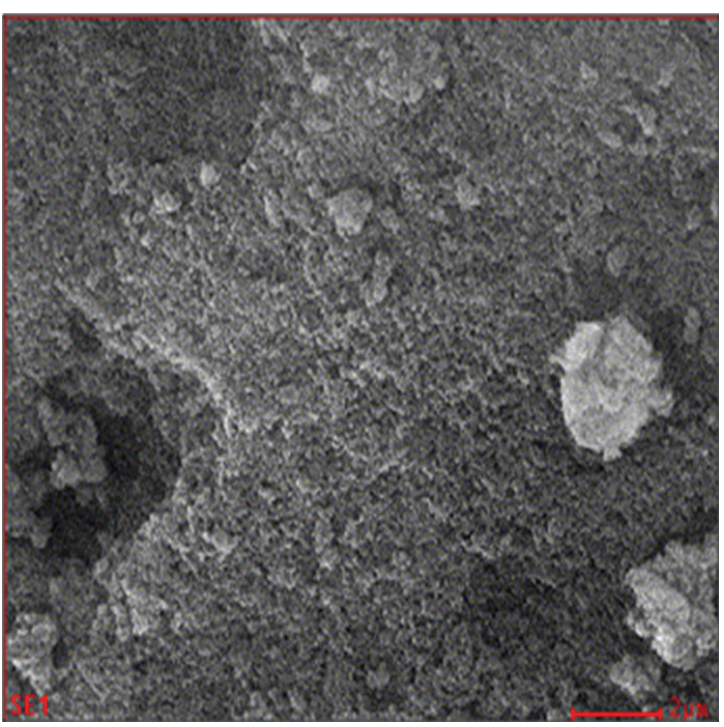

(b)

FIGURE 3: EDX and SEM image of 20 mol\% Ce loaded MCM-41 material.

2.6. Reusability. After completion of the reaction, the catalyst recovered by simple filtration from the reaction mixture was repeatedly washed with ethanol and the catalyst was reused after activation for successive experiments under similar reaction conditions.

\section{Results and Discussion}

Biginelli reaction deals with the condensation of aldehydes and 1,3-dicarbonyl compounds with urea leading to the formation of dihydropyrimidinone (DHPM) derivatives (Scheme 1).

Initially, we have tried to carry out the condensation of benzaldehyde, ethyl acetoacetate, and urea in various organic solvents under reflux conditions using $100 \mathrm{mg}$ of $20 \mathrm{~mol} \%$ of ceric ion loaded MCM-41 as a catalyst. Due to the environmental concerns, we have tried this reaction under solventfree conditions also. Among the different organic solvents studied, no major difference was observed in the yields of the products, but the solvent-free conditions remained to be the best in terms of reaction time and yield. This may be due to the close proximity of substrates in the solid state reactions rather than in solvent medium (Table 1). Biginelli reaction of benzaldehyde, ethyl acetoacetate, and urea under the described reaction conditions did not proceed in the absence of catalyst, whereas in the presence of $10 \mathrm{~mol} \%$ ceric ion loaded MCM-41 as catalyst under classical heating resulted in $39 \%$ of yield even after longer reaction time, indicating that 


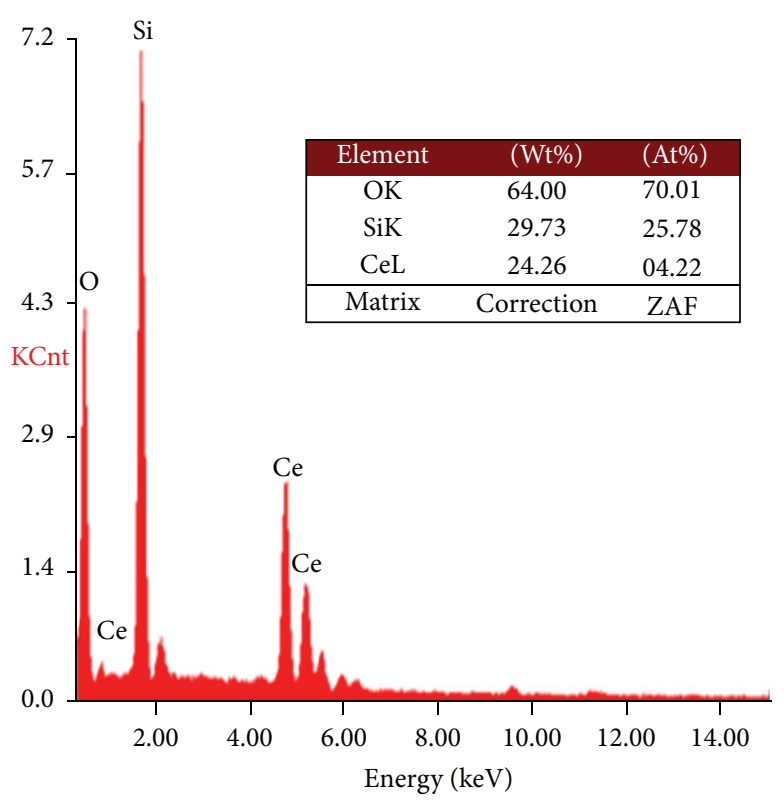

(a)

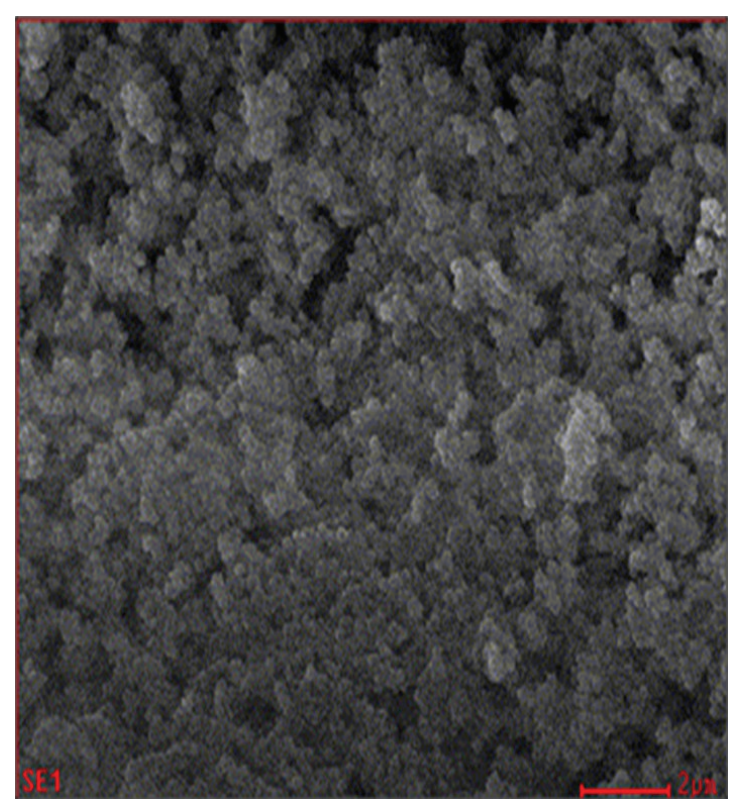

(b)

FIGURE 4: EDX and SEM image of 30 mol\% Ce loaded MCM-41 material.

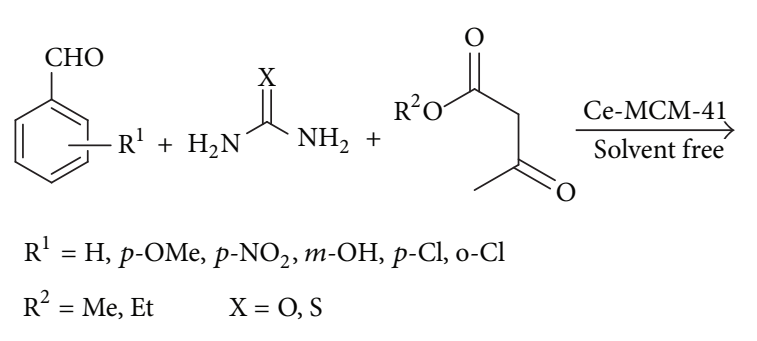<smiles>[R]OC(=O)C1=C(C)NC([X])NC1c1[Y]cccc1</smiles>

Scheme 1: Ce-MCM-41 catalyzed synthesis of DHPMs.

ceric ion is the main promoter for this reaction. Maximum amount of conversion has been achieved by using $20 \mathrm{~mol} \%$ of ceric ion loaded MCM-41 as a

catalyst.

Impressed by the high conversion, we have carried out the above reaction using terephthalaldehyde instead of other monoaromatic aldehydes, with ethyl or methyl acetoacetate and urea or thiourea under same reaction conditions where the yield of about $78-85 \%$ has been achieved (Scheme 2).

The mechanism for Biginelli condensation is well reported in the literature [9]. With supported reagent, the proposed mechanism for the Biginelli reaction may involve the solid acid catalyzed formation of an intermediate of the type I from aldehyde and urea components. Interception of the iminium ion by ethyl acetoacetate produces an open chain ureide II which subsequently cylices to the dihydropyrimidinones III (Figure 5).

3.1. Effect of Reaction Temperature. To evaluate the effect of reaction temperature, Biginelli condensation of benzaldehyde, urea, and ethyl acetoacetate in the presence of Ce-MCM41 was carried out at different temperatures. At room temperature the reaction rate was found to be very slow and was increased with increase in temperature. At $80^{\circ} \mathrm{C}$, the maximum reaction rate was observed and further increase in temperature did not show any significant raise on the yields (Tables 2 and 3).

\subsection{Characterization of Products}

3.2.1. 5-Ethoxycarbonyl-6-phenyl-4-(phenyl)-3,4-dihydropyrimidin-2(1H)-one (4a). Colorless needles; Mp 157-159 ${ }^{\circ} \mathrm{C}$; (Yield 94\%); IR: $\nu_{\max }(\mathrm{KBr}) 3384,3309,3224,3116,3058,3031$, 3006, 2988, 2979, 2960, 2934, 2902, 1703, 1667, 1637, 1600, $1494,1473,1456,1445,1413,1396,1372,1350,1335,1291,1281$, $1267,1247,1187,1159,1130,1099,1030,1014 \mathrm{~cm}^{-1} ;{ }^{1} \mathrm{H}-\mathrm{NMR}$ $\delta_{\mathrm{H}}\left(400 \mathrm{MHz}, \mathrm{DMSO}-\mathrm{d}_{6}\right) 1.10\left(3 \mathrm{H}, \mathrm{t}, \mathrm{CH}_{2} \mathrm{CH}_{3}\right), 3.71(2 \mathrm{H}, \mathrm{q}$, $\left.\mathrm{CH}_{2} \mathrm{CH}_{3}\right), 5.24(1 \mathrm{H}, \mathrm{d}, \mathrm{CH}), 7.27-7.31(3 \mathrm{H}, \mathrm{m}), 7.36-7.43(7 \mathrm{H}$, m), $7.85(1 \mathrm{H}, \mathrm{d}, \mathrm{NH}), 9.28(1 \mathrm{H}, \mathrm{s}, \mathrm{NH})$.

3.2.2. 5-(Ethoxycarbonyl)-4-(p-anisyl)-6-methyl-1,2,3,4-tetrahydropyrimidin-2-one (4b). White solid; Mp 203-204 ${ }^{\circ} \mathrm{C}$; 


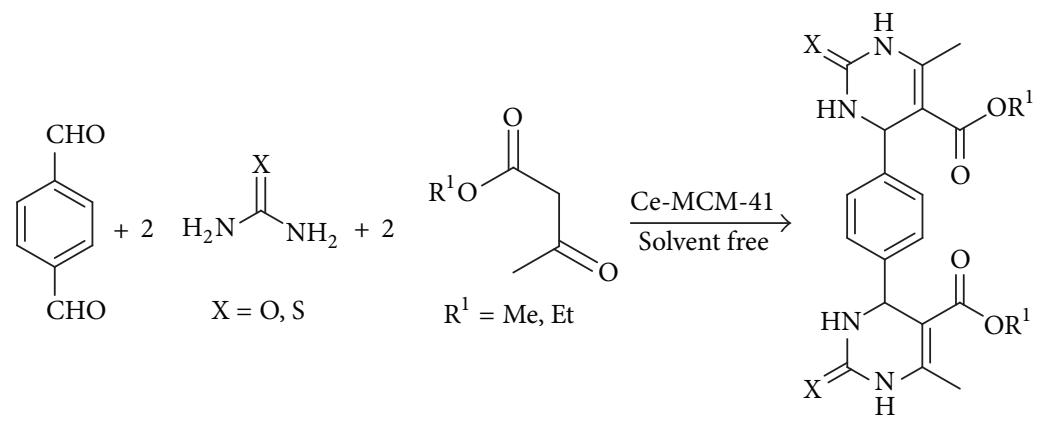

Scheme 2: Ce-MCM-41 catalyzed synthesis of 1,4-phenylene-bis-pyrimidones.

TABLE 1: Synthesis of DHPMs using different catalysts under different reaction conditions ${ }^{a}$.

\begin{tabular}{lcccc}
\hline S. no. & Catalyst & Solvent & Time $(\mathrm{h})$ & Yield $^{\mathrm{b}}(\%)$ \\
\hline 1 & MCM-41 & $\mathrm{EtOH}$ & 6.0 & 44 \\
2 & $\mathrm{SO}_{4}-\mathrm{MCM}-41$ & $\mathrm{EtOH}$ & 4.5 & 48 \\
3 & $\mathrm{Ce}-\mathrm{MCM}-41$ & $\mathrm{MeOH}$ & 4.0 & 61 \\
4 & $\mathrm{Ce}-\mathrm{MCM}-41$ & $\mathrm{EtOH}$ & 3.5 & 64 \\
5 & $\mathrm{Ce}-\mathrm{MCM}-41$ & $\mathrm{CHCl}_{3}$ & 4.0 & 53 \\
6 & $\mathrm{Ce}-\mathrm{MCM}-41$ & $\mathrm{CH}_{3} \mathrm{CN}$ & 4.0 & 58 \\
7 & Ce-MCM-41 & Solvent free & 1.0 & 94 \\
\hline
\end{tabular}

${ }^{\mathrm{a}}$ Condensation of benzaldehyde, EAA, and urea with $20 \mathrm{~mol} \%$ of Ce-MCM-41 (100 mg).

${ }^{\mathrm{b}}$ Isolated yield.

TABLE 2: Ce-MCM-41 catalyzed synthesis of 3,4-dihydropyrimidinones ${ }^{\mathrm{a}}$.

\begin{tabular}{|c|c|c|c|c|c|c|}
\hline S. no. & $R^{1}$ & $R^{2}$ & $X$ & Product & Time (h) & Yield $(\%)^{\mathrm{b}}$ \\
\hline 1 & $\mathrm{H}$ & Et & $\mathrm{O}$ & $4 a$ & 1.0 & 94 \\
\hline 2 & 4-OMe & Et & $\mathrm{O}$ & $4 b$ & 1.5 & 90 \\
\hline 3 & 4-Cl & Et & $\mathrm{O}$ & $4 c$ & 1.0 & 93 \\
\hline 4 & $4-\mathrm{NO}_{2}$ & Et & $\mathrm{O}$ & $4 d$ & 1.5 & 89 \\
\hline 5 & $4-\mathrm{OH}$ & Et & $\mathrm{O}$ & $4 e$ & 1.5 & 88 \\
\hline 6 & $2-\mathrm{OH}$ & Et & $\mathrm{O}$ & $4 f$ & 2.0 & 87 \\
\hline 7 & $2-\mathrm{Cl}$ & Et & $\mathrm{O}$ & $4 \mathrm{~g}$ & 1.5 & 84 \\
\hline 8 & $3-\mathrm{NO}_{2}$ & Et & $\mathrm{O}$ & $4 h$ & 2.0 & 86 \\
\hline 9 & $2-\mathrm{NO}_{2}$ & Et & $\mathrm{O}$ & $4 \mathbf{i}$ & 1.0 & 81 \\
\hline 10 & $\mathrm{H}$ & $\mathrm{Me}$ & $\mathrm{O}$ & $4 j$ & 1.0 & 93 \\
\hline 11 & 4-Cl & $\mathrm{Me}$ & $\mathrm{O}$ & $4 k$ & 1.0 & 90 \\
\hline 12 & $4-\mathrm{NO}_{2}$ & $\mathrm{Me}$ & $\mathrm{O}$ & 41 & 1.5 & 87 \\
\hline 13 & $3-\mathrm{NO}_{2}$ & $\mathrm{Me}$ & $\mathrm{O}$ & $4 \mathrm{~m}$ & 2.0 & 85 \\
\hline 14 & $2-\mathrm{OH}$ & $\mathrm{Me}$ & $\mathrm{O}$ & $4 n$ & 1.5 & 83 \\
\hline 15 & $4-\mathrm{OCH}_{3}$ & $\mathrm{Me}$ & $\mathrm{O}$ & 40 & 2.0 & 89 \\
\hline 16 & $\mathrm{H}$ & Et & $S$ & $4 p$ & 1.0 & 89 \\
\hline 17 & 4-Cl & Et & $\mathrm{S}$ & $4 q$ & 1.5 & 91 \\
\hline 18 & $4-\mathrm{NO}_{2}$ & Et & $S$ & $4 r$ & 1.5 & 86 \\
\hline 19 & $3-\mathrm{NO}_{2}$ & Et & S & $4 s$ & 1.5 & 84 \\
\hline 20 & $\mathrm{H}$ & $\mathrm{Me}$ & $S$ & $4 t$ & 1.0 & 90 \\
\hline 21 & $4-\mathrm{Cl}$ & $\mathrm{Me}$ & $S$ & $4 u$ & 1.0 & 91 \\
\hline 22 & $4-\mathrm{NO}_{2}$ & $\mathrm{Me}$ & S & $4 v$ & 1.5 & 87 \\
\hline
\end{tabular}

${ }^{\mathrm{a}} \mathrm{Ce}-\mathrm{MCM}-41$ catalyzed Biginelli reaction under solvent-free conditions.

${ }^{\mathrm{b}}$ Isolated yields. 
TABLE 3: Ce-MCM-41 catalyzed synthesis of 1,4-phenylene-bis-dihydropyrimidinones ${ }^{\mathrm{a}}$.

\begin{tabular}{lccccc}
\hline S. no. & $R^{1}$ & $X$ & Product & Time (h) & Yield (\%) \\
\hline 1 & Et & O & $\mathbf{5 a}$ & 1.0 & 84 \\
2 & Et & S & $\mathbf{5 b}$ & 1.25 & 80 \\
3 & $\mathrm{Me}$ & $\mathrm{O}$ & $\mathbf{5 c}$ & 1.0 & 82 \\
4 & $\mathrm{Me}$ & $\mathrm{S}$ & $\mathbf{5 d}$ & 1.25 & 79 \\
\hline
\end{tabular}

${ }^{\mathrm{a}} \mathrm{Ce}-\mathrm{MCM}-41$ catalyzed Biginelli reaction under solvent-free conditions.

${ }^{\mathrm{b}}$ Isolated yields.<smiles>[R]C=NC(N)=O</smiles>

Figure 5: Plausible mechanism for the Ce-MCM-41 catalyzed Biginelli reaction.

(Yield 90\%); IR (KBr) v: 3246, 3111, 1709, 1649, 1462, 1285, $1089,787 \mathrm{~cm}^{-1}$; ${ }^{1} \mathrm{H}-\mathrm{NMR} \delta(\mathrm{ppm})\left(400 \mathrm{MHz}, \mathrm{DMSO}-\mathrm{d}_{6}\right): 2.01$ $\left(3 \mathrm{H}, \mathrm{t}, \mathrm{CH}_{3}\right), 2.92\left(3 \mathrm{H}, \mathrm{s}, \mathrm{CH}_{3}\right), 3.91\left(2 \mathrm{H}, \mathrm{q}, \mathrm{CH}_{2}\right), 5.14(1 \mathrm{H}, \mathrm{d}$, $\mathrm{CH}), 6.85(3 \mathrm{H}, \mathrm{m}), 7.23(1 \mathrm{H}, \mathrm{d}), 7.74(1 \mathrm{H}$, br s, NH), $9.15(1 \mathrm{H}, \mathrm{s}$, $\mathrm{NH}) .{ }^{13} \mathrm{C}\left(\mathrm{DMSO}_{-}\right) \delta(\mathrm{ppm}): 165.8,158.9,152.6,148.4,137.5$, $127.8,127.7,114.1,114.0,100.0,59.9,55.5,53.8,18.2,14.5$.

3.2.3. 5-Ethoxycarbonyl-6-methyl-4-(4-chlorophenyl)-3,4-dihydropyrimidin-2(1H)-one $(\mathbf{4 c )}$. White needles; Mp 230232॰C; (Yield 93\%); IR: $\nu_{\max }(\mathrm{KBr}) 3243,3118,2981,1702$, $1648,1575,1490,1461,1422,1367,1323,1292,1220,1182$, $1170,1088,1026,1011 \mathrm{~cm}^{-1}$; ${ }^{1} \mathrm{H}-\mathrm{NMR} \delta(\mathrm{ppm})(400 \mathrm{MHz}$, DMSO-d 6$) 1.09\left(3 \mathrm{H}, \mathrm{t}, \mathrm{CH}_{2} \mathrm{CH}_{3}\right) ; 2.24\left(3 \mathrm{H}, \mathrm{s}, \mathrm{CH}_{3}\right) ; 3.95$ $\left(2 \mathrm{H}, \mathrm{q}, \mathrm{CH}_{2} \mathrm{CH}_{3}\right), 5.13(1 \mathrm{H}, \mathrm{d}, \mathrm{CH}) ; 7.23(2 \mathrm{H}, \mathrm{d}) ; 7.37(2 \mathrm{H}, \mathrm{d})$; 7.77 (1H, br s, NH); 9.24 (1H, br s, NH).

3.2.4. 5-Ethoxycarbonyl-6-methyl-4-(4-nitrophenyl)-3,4-dihydropyrimidin-2(1H)-one (4d). White needles; Mp 216-217 ${ }^{\circ} \mathrm{C}$ (Yield 89\%); IR: $v_{\max }(\mathrm{KBr}) 3353,3228,3113,2979,1697$, $1639,1572,1454,1369,1321,1299,1255,1227,1145,1096$, $1027 \mathrm{~cm}^{-1} ;{ }^{1} \mathrm{H}-\mathrm{NMR}\left(400 \mathrm{MHz}, \mathrm{DMSO}-\mathrm{d}_{6}\right) \delta(\mathrm{ppm}) 1.01(3 \mathrm{H}$, $\left.\mathrm{t}, \mathrm{CH}_{2} \mathrm{CH}_{3}\right), 2.31\left(3 \mathrm{H}, \mathrm{s}, \mathrm{CH}_{3}\right), 4.11\left(2 \mathrm{H}, \mathrm{q}, \mathrm{CH}_{2} \mathrm{CH}_{3}\right), 5.34$ (1H, d, CH), 7.08-7.33 (3H, m, arom.), 7.40 (1H, d, arom.), 7.71 (1H, br s, NH), 9.28 (1H, s, NH). ${ }^{13} \mathrm{C}$ NMR: $\delta(\mathrm{ppm}) 193.96$, $151.93,151.55,149.09,148.97,146.69,127.69,123.83,109.46$, $53.13,53.04,40.33,40.05,39.76,39.50,39.22,38.94,38.67$, $30.65,19.12,19.05$.

3.2.5. Ethoxycarbonyl-6-methyl-4-(2-chlorophenyl)-3,4-di-

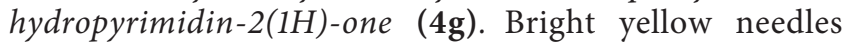

(Yield 84\%); Mp 212-214 ${ }^{\circ}$; IR: $\nu_{\max }(\mathrm{KBr}) 3479,3325,3252$, $3125,2962,1718,1703,1652,1606,1583,1557,1463,1377,1349$, $1319,1287,1223,1173,1142,1089,1014 \mathrm{~cm}^{-1} ; \mathrm{H}^{1}-\mathrm{NMR} \delta_{\mathrm{H}}$ $\left(400 \mathrm{MHz}, \mathrm{DMSO}-\mathrm{d}_{6}\right) 0.74\left(3 \mathrm{H}, \mathrm{t}, \mathrm{CH}_{2} \mathrm{CH}_{3}\right), 2.31(3 \mathrm{H}, \mathrm{s}$, $\left.\mathrm{CH}_{3}\right), 3.69-3.80\left(2 \mathrm{H}, \mathrm{m}, \mathrm{CH}_{2} \mathrm{CH}_{3}\right), 5.29(1 \mathrm{H}, \mathrm{d}, \mathrm{CH}), 7.52$ $(1 \mathrm{H}, \mathrm{d}), 7.58-7.61(1 \mathrm{H}, \mathrm{m}), 7.90(1 \mathrm{H}$, br s, NH), 8.21-8.24 $(2 \mathrm{H}$, m), $9.38(1 \mathrm{H}, \mathrm{s}, \mathrm{NH})$.

3.2.6. 5-Ethoxycarbonyl-6-methyl-4-(3-nitrophenyl)-3,4-dihydropyrimidin-2(1H)-one (4h). Yellow solid; Mp 222-224 ${ }^{\circ} \mathrm{C}$; (Yield 86\%); $\mathrm{H}^{1}$-NMR (400 MHz, DMSO-d 6 ) $\delta(\mathrm{ppm}) 1.09$ $\left(3 \mathrm{H}, \mathrm{t}, \mathrm{CH}_{2} \mathrm{CH}_{3}\right), 2.27\left(3 \mathrm{H}, \mathrm{s}, \mathrm{CH}_{3}\right), 4.00\left(2 \mathrm{H}, \mathrm{q}, \mathrm{CH}_{2} \mathrm{CH}_{3}\right)$, $5.30(1 \mathrm{H}, \mathrm{d}, \mathrm{CH}), 7.71(2 \mathrm{H}, \mathrm{m}), 7.91(1 \mathrm{H}, \mathrm{s}), 8.15(1 \mathrm{H}, \mathrm{s}), 8.15$ (1H, br s, NH), $9.28(1 \mathrm{H}, \mathrm{s}, \mathrm{NH})$.

3.2.7. Methyl-6-methyl-2-oxo-4-phenyl-1,2,3,4-tetrahydro pyrimidine-5-carboxylate (4j). White crystal (Yield 93\%), Mp 208-210 C; IR: $v_{\max }(\mathrm{KBr}) 3246,1732,1664 \mathrm{~cm}^{-1} ;{ }^{1} \mathrm{H}-\mathrm{NMR}$ $\left(400 \mathrm{MHz}, \mathrm{DMSO}-\mathrm{d}_{6}\right) \delta(\mathrm{ppm}) 2.2\left(\mathrm{~s}, 3 \mathrm{H},-\mathrm{CH}_{3}\right), 2.6(\mathrm{~s}, 3 \mathrm{H}$, $\left.-\mathrm{COOCH}_{3}\right), 5.32(\mathrm{~d}, 1 \mathrm{H},-\mathrm{CH}), 7.35(\mathrm{~m}, 5 \mathrm{H}), 7.94(\mathrm{~s}, 1 \mathrm{H}$, $\mathrm{H}), 9.30$ (s, 1H, NH). ${ }^{13} \mathrm{C}$ NMR $\delta(\mathrm{ppm}) 194.29,152.15,152.11$, $152.09,148.14,148.02,144.23,128.54,127.36,126.44,109.59$, $53.80,53.70,40.33,40.05,39.78,39.50,39.22,38.94,38.67$, $30.33,18.92,18.85$.

3.2.8. Ethyl-6-methyl-4-phenyl-2-thioxo-1,2,3,4-tetrahydro pyrimidine-5-carboxylate (4p). Colorless needles; $\mathrm{Mp} 149$ $150^{\circ} \mathrm{C}$; (Yield 89\%); ${ }^{1} \mathrm{H}-\mathrm{NMR} \delta_{\mathrm{H}}\left(400 \mathrm{MHz}, \mathrm{DMSO}-\mathrm{d}_{6}\right) 1.10$ $\left(3 \mathrm{H}, \mathrm{t}, \mathrm{CH}_{2} \mathrm{CH}_{3}\right), 3.71\left(2 \mathrm{H}, \mathrm{q}, \mathrm{CH}_{2} \mathrm{CH}_{3}\right), 5.24(1 \mathrm{H}, \mathrm{d}, \mathrm{CH})$, 
7.27-7.31 (3H, m), 7.36-7.43 (7H, m), 7.85 (1H, d, NH), 9.28 $(1 \mathrm{H}, \mathrm{s}, \mathrm{NH})$.

3.2.9. Diethyl-4,4'-(1,4-phenylene)-bis-(6-methyl-2-oxo-1,2,3, 4-tetrahydropyrimidine-5-carboxylate) (5a). Mp $318-320^{\circ} \mathrm{C}$; (Yield 84\%); IR: $v_{\max }(\mathrm{KBr}) 3329,3110,2972,1692,1246 \mathrm{~cm}^{-1}$; ${ }^{1} \mathrm{H}-\mathrm{NMR}\left(400 \mathrm{MHz}, \mathrm{DMSO}-\mathrm{d}_{6}\right) \delta(\mathrm{ppm}) 9.12(\mathrm{sbr}, 2 \mathrm{H}, \mathrm{NH})$, 7.72 (s, 2H, NH), 7.06 (s, 4H, Ar), 5.31 (d, 2H, CH), 3.77 (q, $\left.4 \mathrm{H}, \mathrm{CH}_{2} \mathrm{CH}_{3}\right), 2.21(\mathrm{~s}, 6 \mathrm{H}, \mathrm{Me}), 1.05\left(\mathrm{t}, 6 \mathrm{H}, \mathrm{CH}_{2} \mathrm{CH}_{3}\right) ;{ }^{13} \mathrm{C}$ NMR $\delta(\mathrm{ppm}) 172.2,153.9,149.2,146.7,132.2,100.1,60.1,49.5$, 18.6, 13.9.

3.2.10. Dimethyl-4,4'-(1,4-phenylene)-bis-(6-methyl-2-oxo-1,2, 3,4-tetrahydropyrimidine-5-carboxylate) $(5 \mathrm{c}) . \mathrm{Mp} 314-316^{\circ} \mathrm{C}$; (Yield 82\%); IR: $\nu_{\max }(\mathrm{KBr}) \mathrm{IR}(\mathrm{KBr}): 3228,3119,1694 \mathrm{~cm}^{-1}$; ${ }^{1} \mathrm{H}-\mathrm{NMR}\left(400 \mathrm{MHz}, \mathrm{DMSO}-\mathrm{d}_{6}\right) ; \delta(\mathrm{ppm}) 2.25(\mathrm{~s}, 6 \mathrm{H}), 3.48$ $(\mathrm{s}, 6 \mathrm{H}), 5.17(\mathrm{~d}, 2 \mathrm{H}), 7.09(\mathrm{~s}, 4 \mathrm{H}), 7.68(\mathrm{~s}, 2 \mathrm{H}), 9.21(\mathrm{~s}, 2 \mathrm{H}) .{ }^{13} \mathrm{C}$ NMR $\delta(\mathrm{ppm})$ 8.64, 51.23, 53.19, 125.17, 130.79, 144.63, 147.35, 151.11.

\section{Conclusion}

Synthesis of mono- and bis-dihydropyrimidin-2(1H)-ones (DPHMs) has been achieved using $20 \mathrm{~mol} \%$ of ceric ion loaded MCM-41 as a catalyst under solvent-free conditions. The present method has some distinct advantages in terms of simple manipulation with the use of ordinary laboratory reagents, their inexpensiveness and nontoxic nature, and excellent yields. The above things make the current procedure an attractive addition to existing methodologies.

\section{Acknowledgment}

The authors gratefully acknowledge the financial support from UGC Minor Research Project F. no. MRP/SERO/3817/ 11, Hyderabad, India.

\section{References}

[1] Zhu and H. Bienayme, Multi-Component Reactions, John Wiley \& Sons, Weinheim, Germany, 2005.

[2] A. Hasaninejad, A. Zare, M. Shekouhy, and J. Ameri Rad, "Catalyst-free one-pot four component synthesis of polysubstituted imidazoles in neutral ionic liquid 1-butyl-3-methylimidazolium bromide," Journal of Combinatorial Chemistry, vol. 12, no. 6, pp. 844-849, 2010.

[3] M. A. Zolfigol, A. Khazaei, A. R. Moosavi-Zare, A. Zare, and V. Khakyzadeh, "Rapid synthesis of 1-amidoalkyl-2-naphthols over sulfonic acid functionalized imidazolium salts," Applied Catalysis A, vol. 400, no. 1-2, pp. 70-81, 2011.

[4] L. Weber, K. Illgen, and M. Almstetter, "Discovery of new multicomponent reactions with combinatorial methods," Synlett, vol. 3, pp. 366-374, 1999.

[5] H. Bienayme, C. Hulme, G. Oddon, and P. Schmitt, "Maximizing synthetic efficiency: multi-component transformations lead the way," Chemistry A, vol. 6, no. 18, pp. 3321-3329, 2000.

[6] N. K. Terrett, Combinatorial Chemistry, Oxford University Press, New York, NY, USA, 1998.
[7] A. Domling, "Recent developments in isocyanide based multicomponent reactions in applied chemistry," Chemical Reviews, vol. 106, pp. 17-89, 2006.

[8] A. Kumar, S. Sharma, R. A. Maurya, and J. Sarkar, "Diversity oriented synthesis of benzoxanthene and benzochromene libraries via one-pot, three-component reactions and their antiproliferative activity," Journal of Combinatorial Chemistry, vol. 12, no. 1, pp. 20-24, 2010.

[9] P. Biginelli, "Derivati aldeiduredici degli eteri acetil-e dossalacetico," Gazzetta Chimica Italiana, vol. 23, pp. 360-416, 1893.

[10] C. O. Kappe, "Biologically active dihydropyrimidones of the Biginelli-type-a literature survey," European Journal of Medicinal Chemistry, vol. 35, no. 12, pp. 1043-1052, 2000.

[11] E. W. Hurst and R. Hull, "Two new synthetic substances active against viruses of the psittacosis-lymphogranuloma-trachoma group," Journal of Medicinal and Pharmaceutical Chemistry, vol. 3, no. 2, pp. 215-229, 1961.

[12] M. Ashok, B. S. Holla, and N. S. Kumari, "Convenient one pot synthesis of some novel derivatives of thiazolo[2,3b]dihydropyrimidinone possessing 4-methylthiophenyl moiety and evaluation of their antibacterial and antifungal activities," European Journal of Medicinal Chemistry, vol. 42, no. 3, pp. 380385, 2007.

[13] S. W. Fewell, C. M. Smith, M. A. Lyon et al., "Small molecule modulators of endogenous and co-chaperone-stimulated Hsp70 ATPase activity," Journal of Biological Chemistry, vol. 279, no. 49, pp. 51131-51140, 2004.

[14] C. I. Carswell, G. L. Plosker, and B. Jarvis, “Rosuvastatin,” Drugs, vol. 62, no. 14, pp. 2075-2085, 2002.

[15] E. H. Hu, D. R. Silder, and U. H. Dolling J, "Unprecedented catalytic three component one-pot condensation reaction: an efficient synthesis of 5-alkoxycarbonyl-4-aryl-3,4dihydropyrimidin-2(1H)-ones," The Journal of Organic Chemistry, vol. 63, no. 10, pp. 3454-3457, 1998.

[16] Y. Ma, C. T. Qian, L. M. Wang, and M. Yang, "Lanthanide triflate catalyzed biginelli reaction. One-pot synthesis of dihydropyrimidinones under solvent-free conditions," The Journal of Organic Chemistry, vol. 65, no. 12, pp. 3864-3868, 2000.

[17] A. Brindban and J. U. Jana, "Indium (III) chloride-catalyzed one-pot synthesis of dihydropyrimidinones by a threecomponent coupling of 1,3-dicarbonyl compounds, aldehydes, and urea: an improved procedure for the biginelli reaction," The Journal of Organic Chemistry, vol. 65, no. 19, pp. 6270-6272, 2000.

[18] G. Salitha, K. B. Reddy, and J. S. Yadav, "Vanadium(III) chloride catalyzed Biginelli condensation: solution phase library generation of dihydropyrimidin-(2H)-ones," Tetrahedron Letters, vol. 44, no. 34, pp. 6497-6499, 2003.

[19] M. Gohain, D. Prajapati, and J. S. Sandhu, "A novel cu-catalysed three-component one-pot synthesis of dihydropyrimidin$2(1 \mathrm{H})$-ones using microwaves under solvent-free conditions," Synlett, no. 2, pp. 235-238, 2004.

[20] G. Maiti, P. Kundu, and C. Guin, "One-pot synthesis of dihydropyrimidinones catalysed by lithium bromide: an improved procedure for the biginelli reaction," Tetrahedron Letters, vol. 44, no. 13, pp. 2757-2758, 2003.

[21] C. V. Reddy, M. Mahesh, P. V. K. Raju, T. R. Babu, and V. V. N. Reddy, "Zirconium(IV) chloride catalyzed one-pot synthesis of 3,4-dihydropyrimidin-2(1H)-ones," Tetrahedron Letters, vol. 43, no. 14, pp. 2657-2659, 2002. 
[22] J. S. Yadav, B. V. Subba Reddy, R. Srinivas, C. Venugopal, and T. Ramalingam, "LiClO4-catalyzed one-pot synthesis of dihydropyrimidinones: an improved protocol for Biginelli reaction," Synthesis, no. 9, pp. 1341-1345, 2001.

[23] A. Dondoni and A. Massi, "Synthetic studies toward the microtubule-stabilizing agent laulimalide: synthesis of the $\mathrm{C}_{1}$ $\mathrm{C}_{14}$ fragment," Tetrahedron Letters, vol. 42, no. 5, pp. 797-800, 2001.

[24] T. Jin, S. Zhang, and T. Li, " $p$-Toluenesulfonic acid-catalyzed efficient synthesis of dihydropyrimidines: improved high yielding protocol for the biginelli reaction," Synthetic Communications, vol. 32, no. 12, pp. 1847-1851, 2002.

[25] P. Salehi, M. Dabiri, M. A. Zolfigol, and M. A. Bodaghi Fard, "Silica sulfuric acid: an efficient and reusable catalyst for the one-pot synthesis of 3,4-dihydropyrimidin-2(1H)-ones," Tetrahedron Letters, vol. 44, no. 14, pp. 2889-2891, 2003.

[26] S. Tu, F. Fang, S. Zhu, T. Li, X. Zhang, and Q. Zhuang, "A new biginelli reaction procedure using potassium hydrogen sulfate as the promoter for an efficient synthesis of 3,4dihydropyrimidin-2(1H)-one," Synlett, no. 3, pp. 537-539, 2004.

[27] F. Bigi, S. Carloni, B. Frullanti, R. Maggi, and G. Sartori, "A revision of the biginelli reaction under solid acid catalysis. Solvent-free synthesis of dihydropyrimidines over montmorillonite KSF," Tetrahedron Letters, vol. 40, no. 17, pp. 3465-3468, 1999.

[28] M. Tajbakhsh, B. Mohajerani, M. M. Heravi, and A. N. Ahmadi, "Natural HEU type zeolite catalyzed Biginelli reaction for the synthesis of 3,4-dihydropyrimidin-2(1H) one derivatives," Journal of Molecular Catalysis A, vol. 236, no. 1-2, pp. 216-219, 2005.

[29] V. Radha Rani, N. Srinivas, M. Radha Kishan, S. J. Kulkarni, and K. V. Raghavan, "Zeolite-catalyzed cyclocondensation reaction for the selective synthesis of 3,4-dihydropyrimidin-2(1H)-ones," Green Chemistry, vol. 3, no. 6, pp. 305-306, 2001. 

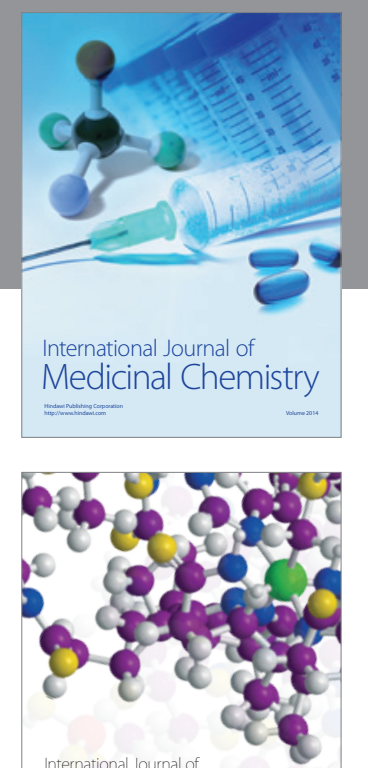

\section{Carbohydrate} Chemistry

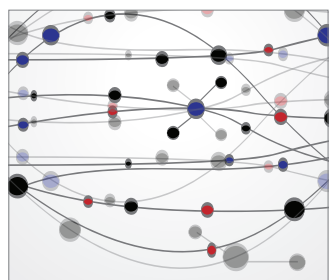

The Scientific World Journal
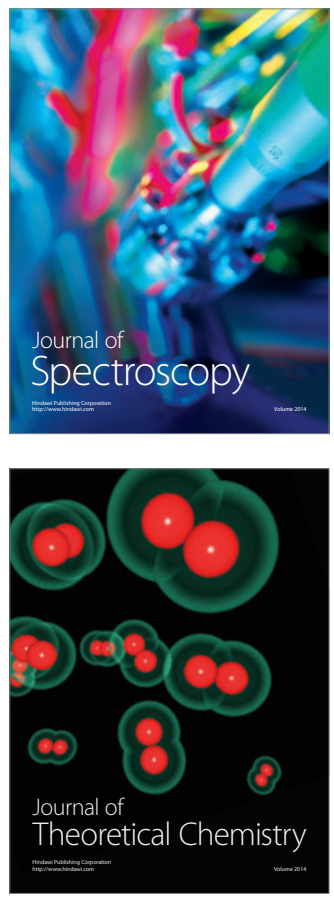
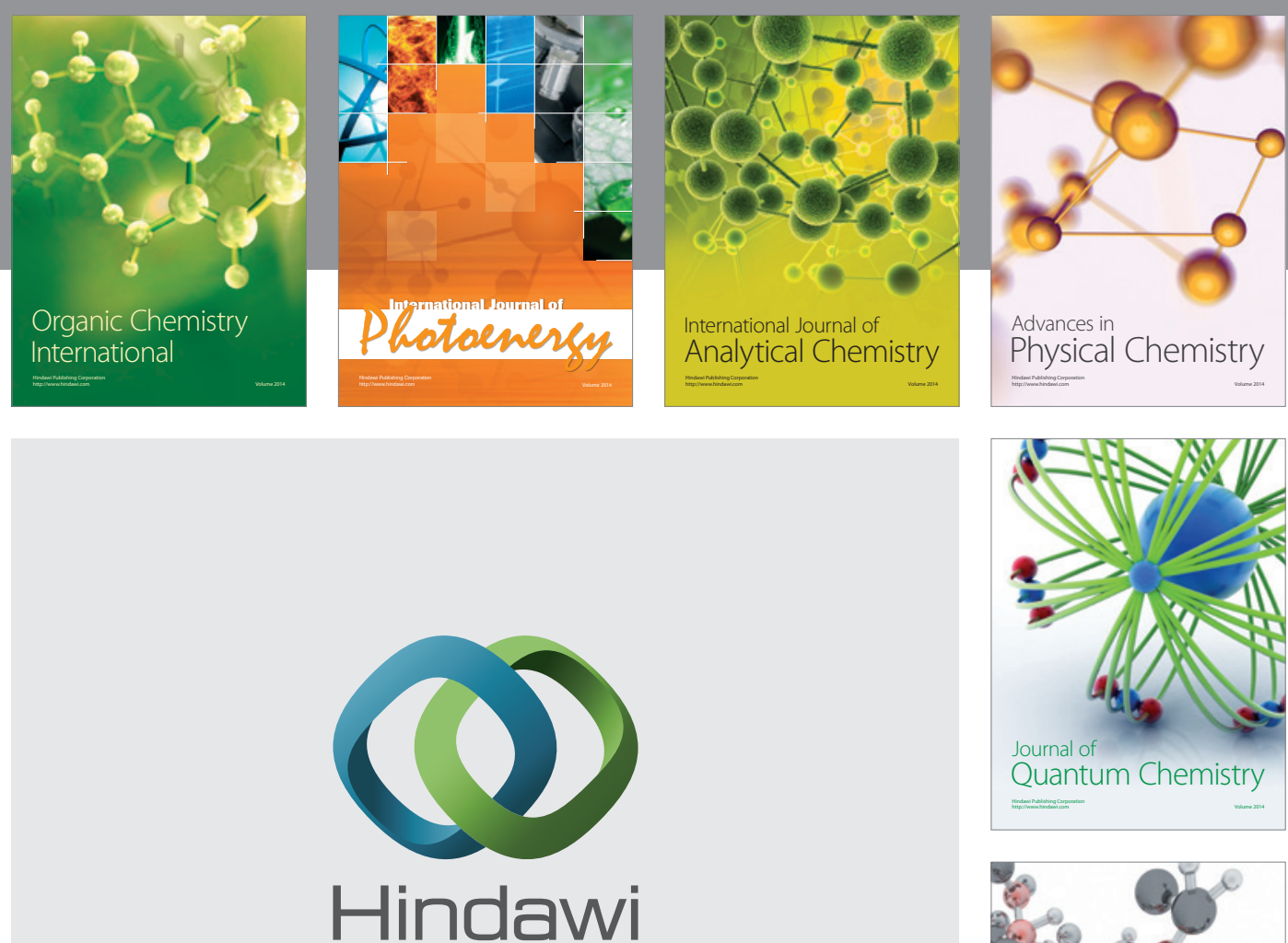

Submit your manuscripts at

http://www.hindawi.com

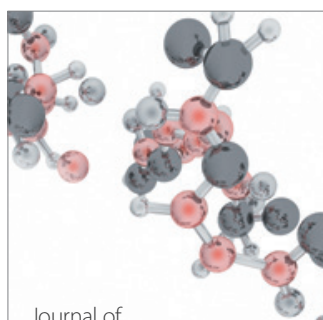

Analytical Methods

in Chemistry

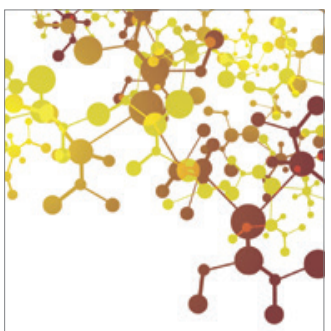

Journal of

Applied Chemistry

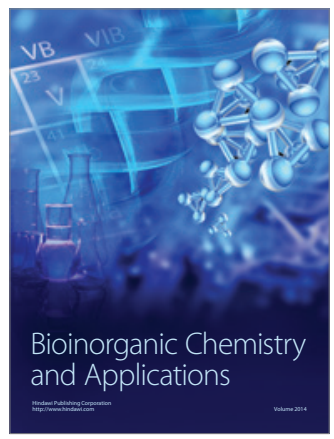

Inorganic Chemistry
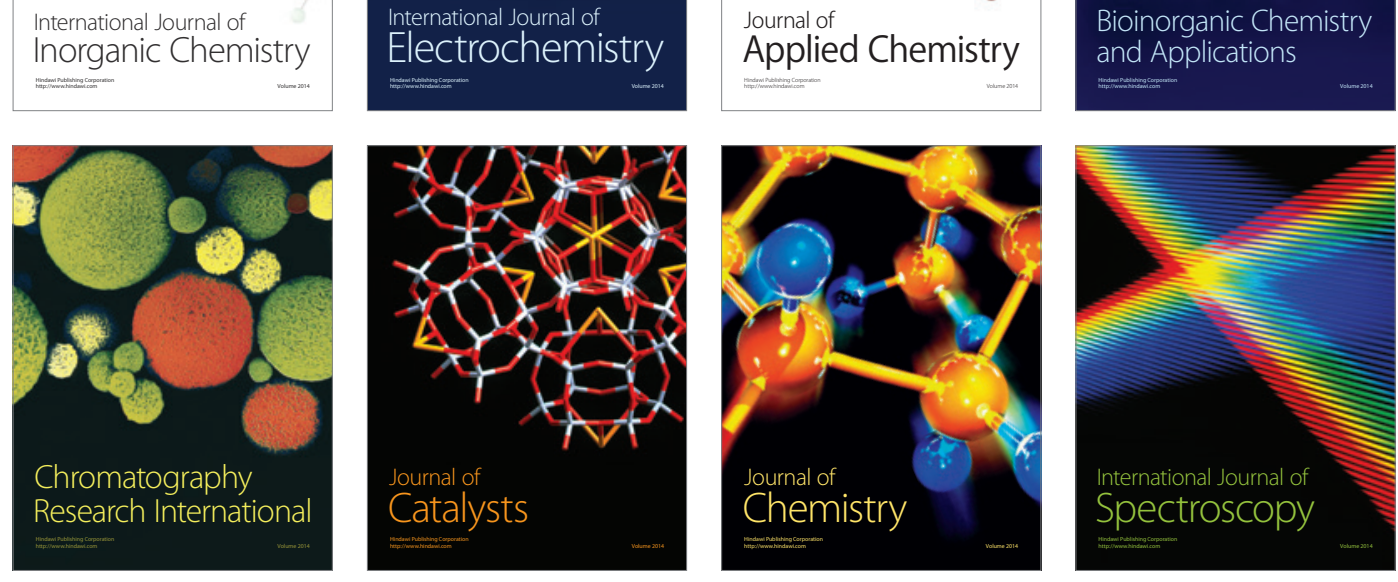